\title{
Dysregulation of PTEN caused by the underexpression of microRNA-130b is associated with the severity of lupus nephritis
}

\author{
SHUPENG WU ${ }^{1}$, JING WANG ${ }^{2}$ and FANG LI ${ }^{1}$ \\ Departments of ${ }^{1}$ Rheumatism and Immunology, and ${ }^{2}$ Geriatric Diseases, Tai'an Central Hospital, \\ Tai'an, Shandong 271000, P.R. China
}

Received February 3, 2016; Accepted August 31, 2017

DOI: $10.3892 / \mathrm{mmr} .2018 .8839$

\begin{abstract}
There are several reports in the literature regarding microRNA (miR)-130b. It has been reported that miR-130b is involved in several diseases. The present study aimed to understand the association between the levels of miR-130b and lupus nephritis in patients. A total of 61 blood samples were collected and the expression level of miR-130b was determined. The online miRNA database was then searched using the 'seed sequence' located within the 3'-untranslated region of the target gene. Linear analysis and a luciferase assay were performed to understand the regulatory association between miR-130b and phosphatase and tensin homolog (PTEN). In addition, reverse transcription-polymerase chain reaction and western blot analyses were performed to examine the mRNA and protein expression levels of PTEN among individuals with lupus nephritis $(\mathrm{n}=28)$ and those without lupus nephritis $(n=31)$, and in mesangial cells treated with scramble control, miR-130b mimics, PTEN small interfering (si)RNA and miR-130b inhibitors. In addition mesangial cells were treated with scramble control, miR-130b mimics, PTEN siRNA and miR-130b inhibitors to investigate the affect of miR-130b and PTEN on the viability and apoptosis of mesangial cells. The results demonstrated that $\mathrm{miR}-130 \mathrm{~b}$ was downregulated in the hormone-resistant group of lupus nephritis patients. PTEN was a virtual target of miR-130b. There was a negative regulatory association between miR-130b and PTEN. The mRNA and protein expression levels of PTEN were increased in the hormone-resistant group. miR-130b decreased the expression of PTEN. miR-130b negatively interfered with the viability of mesangial cells and PTEN positively interfered with the viability of mesangial cells. miR-130b accelerated apoptosis and PTEN inhibited apoptosis. Taken together, the results showed that miR-130b was upregulated in the lupus nephritis
\end{abstract}

Correspondence to: Dr Shupeng Wu, Department of Rheumatism and Immunology, Tai'an Central Hospital, 29 Longtan Road, Tai'an, Shandong 271000, P.R. China

E-mail: lupusnephritis@163.com

Key words: phosphatase and tensin homolog, microRNA-130b, severity, lupus nephritis, apoptosis group. PTEN was a virtual target of miR-130b, and there was a negative regulatory association between miR-130b and PTEN. miR-130b and PTEN interfered with the viability and apoptosis of the mesangial cells.

\section{Introduction}

In total of $\sim 0.1 \%$ of the population are affected by systemic lupus erythematosus (SLE), which is a chronic autoimmune disease involving multiple systems, with women having a nine times higher risk of developing the disease, compared with men (1). SLE is a complex disease, which has a range of manifestations and the combinations of which can lead to variable levels of disease severity. Autoreactive B and T lymphocytes have been the focus of the majority of studies performed to investigate the pathophysiology of this disease (2).

It is a general agreement that clinically relevant nephritis occurs in $60 \%$ of patients with lupus at some point during the duration of the disease (3). It is important that diagnosis is made early and that the treatment of renal disease occurs early, as early response to therapy is associated with more favorable results (4).

Current understanding of the correlation between gene messenger RNAs (mRNAs) and human disease has changed following the identification of microRNAs (miRNAs) at the turn of the 21st century, which marked a new era of cell biology and has extended to those sequences in the residual $\sim 90 \%$ of eukaryotic genomes that produce non-coding RNAs (5). miRNAs function as meta-controllers of gene expression and are pivotal for the cellular alterations required for development (5).

miRNAs are implicated in the pathogenesis of renal fibrosis and chronic kidney diseases. Certain miRNAs exhibit antifibrotic effects and others have profibrotic effects. miR-29 and miR-200 are reduced, whereas miR-21, miR-377, miR-205, miR-141 and miR-192 are elevated in animal models and patients with renal fibrosis, contributing to hypertensive nephrosclerosis, Ig A nephropathy, obstructive nephropathy and diabetic nephropathy (6-10). Additionally, the suppression of miR-192 alleviates renal fibrosis in mice with diabetes (11), and it has been demonstrated that miRNAs serve as crucial mediators in renal fibrosis and may be promising targets for the prevention of end-stage renal disease (12). Few studies have focused on miRNAs in lupus nephritis. In a previous 
study, 66 miRNAs differentially expressed in renal biopsies were identified from patients with lupus nephritis, compared with normal subjects. In another study, there were differences in the intrarenal expression levels of miR-146a, miR-198 and miR-638 between patients with lupus nephritis and normal subjects (13). There have been no reports on the potential of miRNAs as markers of any specific histologic presentation or their possible effect in renal fibrosis in lupus nephritis (14).

Patients with renal failure may have significantly decreased circulating miRNAs, compared with patients with marginal renal impairment or normal renal function, as shown by microarrays. The underlying mechanism may be associated with the substantial buildup of RNase in patients with renal failure, which elevates degraded circulating miRNAs, however, this remains to be elucidated (15). This was demonstrated by the fact that miR-1233-3p and miR-130b were downregulated, respectively, and that the total RNA level was significantly reduced in late stage lupus nephritis of the validation group, which were consistent with the results reported by Neal et al, who determined the serum levels of five specific miRNAs in patients who suffered from renal failure (16).

It has been previously shown that miR-130b is differentially expressed in lens epithelial cells collected from individuals with lupus nephritis, and the dysregulation of PTEN has also been reported to be involved in the molecular mechanism of mesangial cell apoptosis $(17,18)$. By searching an online miRNA database, the present study identified PTEN as a virtual target of miR-133b. It was then confirmed that PTEN was a target of miR-130b, and the involvement of miR-130b and PTEN in the development of lupus nephritis was confirmed.

\section{Materials and methods}

Sample collection. Tissue samples from 28 patients with SLE and lupus nephritis (33 \pm 4 -years old) and 31 healthy patients

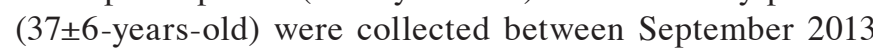
and September 2014 at the Department of Rheumatism and Immunology, Tai'an Central Hospital (Shenyang, China). All patients were assessed by biopsy to confirm fulfillment to the 1982 American College of Hematology revised criteria for SLE and lupus nephritis (19). Written consent was signed by all patients, glomerular filtration rate was estimated using the modification of diet in renal disease formula (20), and clinical and demographic data of the patients were carefully recorded. The protocol of the present study was approved by the Ethics Committee of the Hospital of Tai'an Central Hospital.

Target prediction and functional analysis. By scanning the most commonly used target gene prediction databases, including miRDB (http://www.mirdb.org/), miRanda (http://www.microrna.org/microrna/home.do) and TargetScan (www.targetscan.org), the putative target genes of the miR-130b were pooled from the three databases in total. Experimental validation on most of the target genes was performed. Gene ontology (GO) term analysis were performed to gain a full insight of the functional relevance on these target genes. GO term analysis indicated that these target genes were enriched in critical biological processes, including 'cell proliferation' and 'regulation of focal adhesion kinase activity'.
RNA isolation and reverse transcription-polymerase chain reaction $(R T-P C R)$ analysis of PTEN $m R N A$ and $m i R-130 b$ in tissue samples and mesangial cells. TRIzol extraction reagent (Thermo Fisher Scientific, Inc., Waltham, MA, USA) was used to extract the total RNA from tissue samples and mesangial cells (obtained from the tissue samples) according to manufacturer's protocol. An A260/A280 value between 1.8 and 2.0 was accepted, which ensured that the RNA samples were without DNA or proteins. A TaqMan MicroRNA Reverse Transcription kit (Applied Biosystems; Thermo Fisher Scientific, Inc.) was used to synthesize the cDNA (PTEN) from RNA with a mixture of $2 \mu \mathrm{g}$ total RNA, $1 \mu \mathrm{g}$ of miRNA-specific primers (25 $\mu \mathrm{M})$ and $\mathrm{ddH}_{2} \mathrm{O}$ (RNase-free) to a final volume of $10 \mu \mathrm{l}$. The mixture was then denatured for $10 \mathrm{~min}$ at $70^{\circ} \mathrm{C}$ and placed on ice, followed by the addition of the reaction buffer

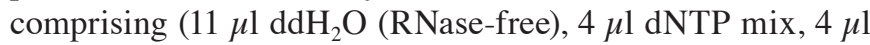
5X RT buffer and $1 \mu \mathrm{l}$ ReverTra Ace (100 U/ $\mu$ l; Toboyo Co., Ltd., Osaka, Japan), and then maintained for $60 \mathrm{~min}$ at $42^{\circ} \mathrm{C}$, followed by $10 \mathrm{~min}$ at $90^{\circ} \mathrm{C}$. Invitrogen Platinum SYBR-Green qPCR SuperMix-UDG (Thermo Fisher Scientific, Inc.) was used to amplify the cDNA according to the manufacturer's protocol with a mixture of $1.5 \mu \mathrm{l}$ forward primer $(10 \mu \mathrm{l}), 25 \mu \mathrm{l}$ SYBR mix, $1.5 \mu \mathrm{l}$ reverse primer $(10 \mu \mathrm{M}), 1 \mu \mathrm{l} \mathrm{cDNA}$ and $21 \mu \mathrm{lddH_{2 }}$ O. Sequences of primers were: miR-130b forward, 5'GGGCAGTGCAATGATGAAA3', reverse, 5'GTGCGTGTC GTGGAGTCG3'; PTEN forward, 5'-TTTGAAGACCATAAC CCACCAC-3', reverse, 5'-ATTACACCAGTTCGTCCCTTT C-3' and GAPDH forward, 5'-AGCCTCAAGATCATCAGC AATG-3' and reverse, 5'-TGTGGTCATGAGTCCTTCCAC G-3'. Fluorescence detection and qPCR were performed using the Qiagen Rotor-Gene Q (Qiagen GmbH, Hilden, Germany). The reaction settings were as follows: $10 \mathrm{~min}$ at $50^{\circ} \mathrm{C}, 10 \mathrm{~min}$ at $95^{\circ} \mathrm{C}, 15 \mathrm{sec}$ at $95^{\circ} \mathrm{C}, 45 \mathrm{sec}$ at $60^{\circ} \mathrm{C}$ for 40 cycles. The $2^{-\Delta \Delta \mathrm{Cq}}$ method (21) was used to calculate the relative mRNA expression of PTEN and miR-130b according to the expression of GAPDH for human renal biopsies. Three assessments were performed in triplicate.

Cell culture and transfection. DMEM/F12 (Gibco; Thermo Fisher Scientific, Inc.) with $1 \%$ penicillin/streptomycin and $10 \%$ fetal bovine serum (FBS; Gibco; Thermo Fisher Scientific, Inc.) was used for incubation of the mesangial cells at $37^{\circ} \mathrm{C}$ in $5 \% \mathrm{CO}_{2}$ until use. When confluence reached $30-50 \%$ and the cells were serum-deprived for $24 \mathrm{~h}$, Lipofectamine ${ }^{\circledR}$ RNAiMAX Transfection reagent (Applied Biosystems; Thermo Fisher Scientific, Inc.) was used to transfect the cells with miR-130b mimics or PTEN small interfering (si)RNA (Sunbio, Shanghai, China) or inhibitors according to protocols provided by the manufacturers. Sequence of PTEN siRNA was as follows: 5'-GGCGUAUACAGGAACAAUATT-3'. The medium was replaced with DMEM/F12 culture medium containing $2 \%$ reduced FBS $6 \mathrm{~h}$ following transfection. The experiments were repeated three times independently.

Cell proliferation assay. An MTT assay was used to assess the cell proliferation, as described previously. The mesangial cells were incubated in a 96 -well plate at a final density of $5 \times 10^{4}$ cells per well for 1 day at $37^{\circ} \mathrm{C}$. Subsequently, $200 \mu \mathrm{l}$ of MTT solution was used to treat the mesangial cells for $60 \mathrm{~min}$ at $37^{\circ} \mathrm{C}$, following which the MTT solution was carefully removed and 
the cells were washed with $100 \mu 1$ PBS. Finally, the cells were treated with $200 \mu \mathrm{l}$ dimethyl sulfoxide solution for $120 \mathrm{~min}$ on a plate shaker at room temperature. A microplate reader (Synergy HT; BioTek, Instruments, Inc., Winooski, VT, USA) was used to measure the proliferation of the mesangial cells based on the absorbance at $575 \mathrm{~nm}$. All tests were performed in triplicate.

Luciferase assay. The 3'-untranslated region (UTR) of PTEN containing the binding site of miR-130b was amplified by PCR and inserted into the psiCHECK-2 reporter vector (Promega Corporation, Madison, WI, USA). The mutagenesis was performed for the same site and introduced into the control vector (Ambion; Thermo Fisher Scientific, Inc.). For transfection, the cells were co-transfected with wild-type/mutant type vector and $\mathrm{miR}-130 \mathrm{~b}$ mimic/negative control using Lipofectamine ${ }^{\circledR} 2000$ (Thermo Fisher Scientific, Inc.) with a mixture of $1 \times 10^{6}$ cells, $1 \mu \mathrm{g}$ Renilla luciferase expression construct, pRL-TK (Promega Corporation), $1 \mu \mathrm{g}$ of psiCHECK-2 reporter vector (Promega Corporation) or psiCHECK-2-mut plasmid and 50 pmol of miR-130b mimic or control. Renilla luciferase activity was used as an internal control. After incubation at $37^{\circ} \mathrm{C}$ for $48 \mathrm{~h}$, the Dual Luciferase assay reagent (Promega Corporation) was used to measure the luciferase activity in accordance with the manufacturer's protocol. All results were calculated as fold differences relative to Renilla luciferase activity. Each experiment was performed at least three times.

Western blot analysis. For analysis of the mRNA expression of PTEN and miR-130b, ice-cold lysis buffer containing $1 \%$ NP-40, 0.1\% sodium dodecyl sulfate, $50 \mathrm{mM}$ Tris- $\mathrm{HCl}$ ( $\mathrm{pH} 7.4$ ) and $150 \mathrm{mM} \mathrm{NaCl}$, including protease inhibitors (Roche Diagnostics, Basel, Switzerland) was used to lyse the mesangial cells according to the manufacturer's protocol. The lysates were centrifuged at $14,000 \mathrm{x}$ g at $4^{\circ} \mathrm{C}$ for $15 \mathrm{~min}$. A Bradford protein assay (Bio-Rad Laboratories, Inc., Hercules, CA, USA) was used to determine the concentration of protein. To separate $35 \mu \mathrm{g}$ protein, $8 \%$ SDS-PAGE was used, and the protein then was transferred onto a polyvinylidene fluoride membrane (EMD Millipore, Billerica, MA, USA) for $60 \mathrm{~min}$ $(120 \mathrm{~V})$. Tris-buffered saline containing $0.1 \%$ Tween-20 (TBST) and 5\% non-fat dry milk was used to block the membrane to avoid unspecific binding. Primary antibodies, including anti- $\beta$-actin (1:5,000; A5441; Sigma-Aldrich; Merck KGaA, Darmstadt, Germany) and anti-PTEN (1:1,000; SAB4300337; Sigma-Aldrich; Merck KGaA) were used to treat the membrane. Subsequently, HRP-conjugated secondary antibody (1:10,000; A6154; Sigma-Aldrich; Merck KGaA) was used for incubation of the membrane. Quantity One 1-D Analysis Software (v4.6.9; Bio-Rad Laboratories, Inc.) was used to quantify the band intensity.

Analysis of apoptosis. Apoptosis was performed using propidium iodide/annexin $\mathrm{V}$ staining with an apoptosis detection kit (Nanjing KeyGEN Biotech Co., Ltd., Nanjing, China). The mesangial cells were maintained at room temperature for 15 min in the dark, following which flow cytometry (BD Biosciences, San Jose, CA, USA) was used to assess the specimens. Annexin V immunofluorescence is shown on the
$\mathrm{X}$-axis and plasma membrane integrity is shown on the Y-axis. Analyses were repeated three times.

Statistical analysis. All data are shown as the mean \pm standard error of the mean. Each experiment was performed at least three times to ensure the reproducibility of each test. One-way analysis of variance or a Student's t-test was used to analyze differences to determine statistical significance between groups. Pearson's linear correlation analysis was used to analyze the correlation between two variables. The predictive accuracy of renal miR-130b levels was assessed using ROC analysis. $\mathrm{P}<0.05$ was considered to indicate a statistically significant difference. SPSS 13.0 (SPSS, Inc., Chicago, IL, USA) was employed for statistical analysis. All tests were repeated three times.

\section{Results}

miR-130 is upregulated in patients with lupus nephritis. In the present study, kidney tissue samples were collected from patients with SLE. These samples were then divided into two groups based on whether they had nephritis or not: Lupus nephritis(+) and lupus nephritis(-), respectively. RT-qPCR analysis was then performed, and the results showed that the expression level of miR-130b was higher in the lupus nephritis(+) group (Fig. 1). These results indicated that miR-130b is a risk factor for lupus nephritis in patients.

PTEN is virtual target of miR-130b. There is a series of literature on miR-130b, and it has been reported that miR-130b is involved in several diseases. The present study was performed to understand the association between miR-130b levels and lupus nephritis. An online miRNA target prediction tool was used to identify the regulatory gene of miR-130b, which identified PTEN as a candidate target gene of miR-130b with the 'seed sequence' in the 3'UTR (Fig. 2) and three existing binding sites. To confirm the regulatory association between miR-130b and PTEN, a luciferase activity reporter assay was performed in mesangial cells. Only the luciferase activity in the mesangial cells cotransfected with miR-130b and wild-type PTEN 3'UTR was decreased significantly (Fig. 3), whereas mesangial cells cotransfected with miR-130b and mutant PTEN 3'UTR showed comparable luciferase activity to that in the scramble control (Fig. 3). These results confirmed that PTEN was a target of miR-130b in mesangial cells. To further examine the regulatory association between miR-130b and PTEN, the correlation between the expression level of miR-130b and mRNA expression of PTEN was examined among the blood samples $(n=61)$, which showed a negative regulatory association (Fig. 4; $\mathrm{r}=-0.5122 ; \mathrm{P}<0.01$ ).

Determination of expression patterns of miR-130b and PTEN in tissues of different groups. The tissue samples of the lupus nephritis(+) $(n=28)$ and lupus nephritis(-) $(n=33)$ patients were used to further examine the effects on the interaction between miR-130b and the PTEN 3'UTR. By performing RT-qPCR analysis, it was found that the mRNA expression of PTEN (Fig. 5A) was decreased in the lupus nephritis(+) group, compared with that in the lupus nephritis(-) group. The protein expression of PTEN (Fig. 5B) was measured using densitometric analysis, which revealed it was also decreased 


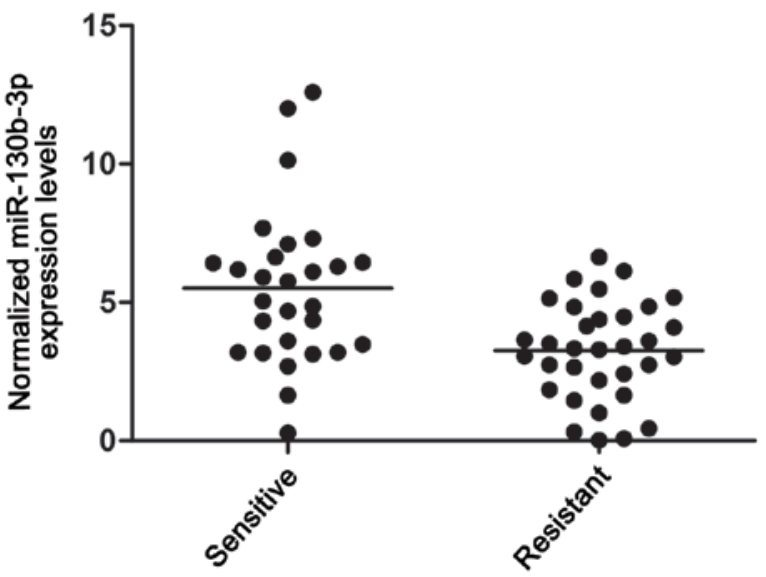

Figure 1. miR-130b is upregulated in patients with lupus nephritis. Expression of miR-130b was higher in the sensitive lupus nephritis(+) cells, compared with that in in the resistant lupus nephritis(-) cells. miR, microRNA.

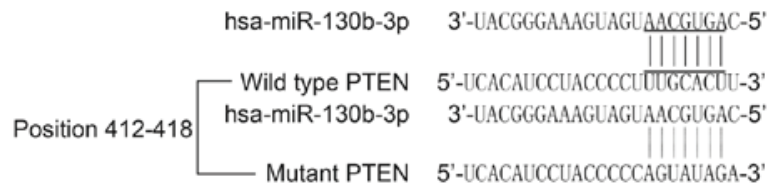

Figure 2. PTEN is a candidate target gene of miR-130b in mesangial cells. The 'seed sequence' was identified in the 3'untranslated region. There were three binding sites. miR, microRNA; PTEN, phosphatase and tensin homolog.

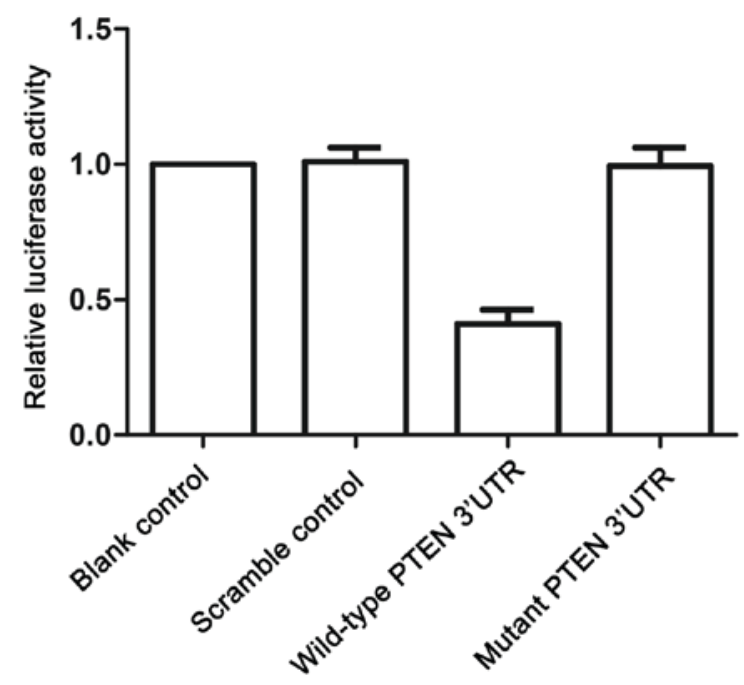

Figure 3. PTEN is a direct target of miR-130b. Luciferase activity reporter assay was performed to verify PTEN as the direct target gene of miR-130b. PTEN, phosphatase and tensin homolog; 3'UTR, 3'untranslated region; miR, microRNA.

in the lupus nephritis(+) group, compared with that in the lupus nephritis(-) group. To further confirm the hypothesis of the negative regulatory association between miR-130b and PTEN, the mesangial cells were transfected with scramble control, miR-130b mimics, PTEN siRNA and miR-130b inhibitors. The protein (Fig. 6A) and mRNA (Fig. 6B) expression levels of PTEN in the mesangial cells treated with iR-130b mimics and PTEN siRNA were lower, compared with those

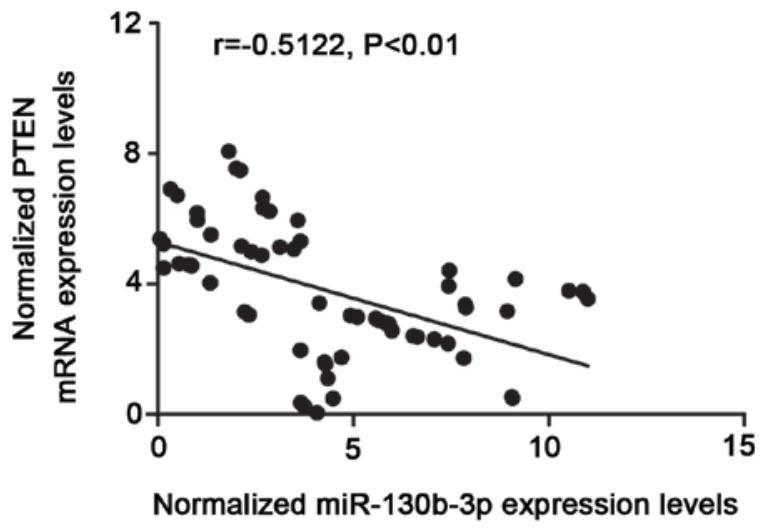

Figure 4. Negative correlation between miR-130b and mRNA expression of PTEN. Correlation between the expression level of miR-130b and mRNA expression of PTEN between the lupus nephritis(+) group $(n=28)$ and lupus nephritis(-) group $(\mathrm{n}=31)$. miR, microRNA; PTEN, phosphatase and tensin homolog.

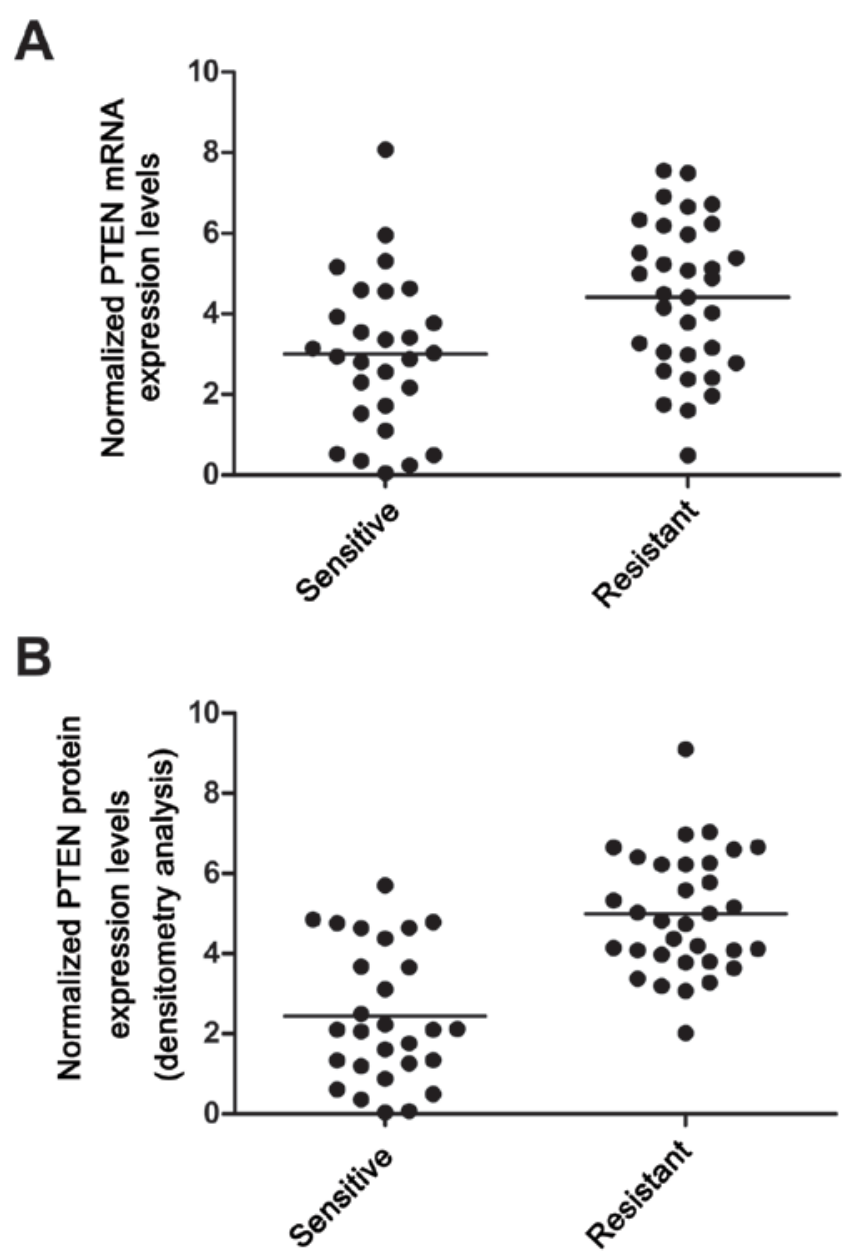

Figure 5. Expression of PTEN is decreased in lupus nephritis. (A) mRNA and (B) protein expression levels of PTEN were decreased in the (sensitive) lupus nephritis(+) group, compared with those in the (resistant) lupus nephritis(-) group. PTEN, phosphatase and tensin homolog.

in the scramble control, whereas mesangial cells treated with miR-130b inhibitors were higher, compared with those in the scramble control. This confirmed the negative regulatory association between miR-130b and PTEN. 
A

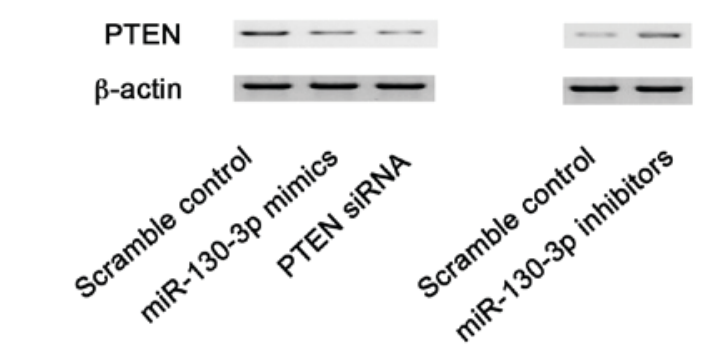

B

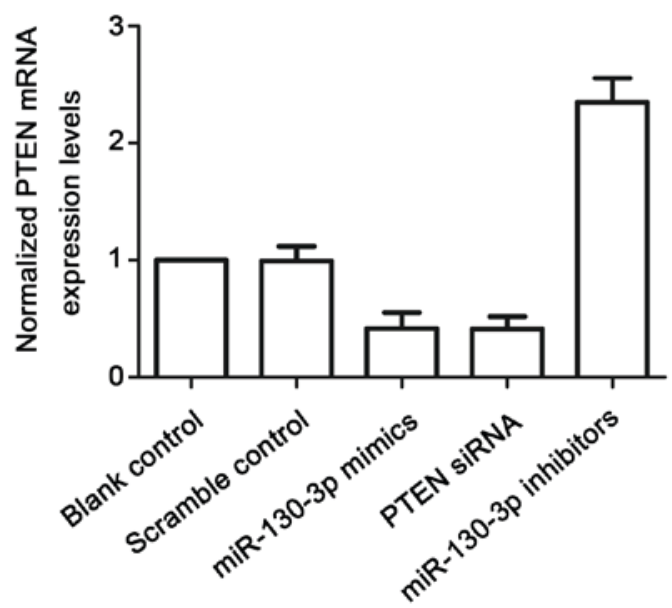

Figure 6. Expression of PTEN in mesangial cells. Mesangial cells were transfected with scramble control, miR-132 mimics, PTEN siRNA and miR-130b inhibitors. (A) The protein and (B) mRNA expression levels of PTEN in mesangial cells treated with miR-130b mimics and PTEN siRNA were decreased, whereas those in mesangial cells treated with miR-130b inhibitors were increased. miR, microRNA; siRNA, small interfering RNA; PTEN, phosphatase and tensin homolog.

miR-130b and PTEN interfere with the viability of mesangial cells. The present study also investigated the relative viability of mesangial cells when transfected with scramble control, miR-130b mimics, PTEN siRNA and miR-130b inhibitors. The mesangial cells transfected with miR-130b inhibitors showed upregulated viability (Fig. 7A), compared with that in the scramble control, whereas the mesangial cells transfected with miR-130b mimics and PTEN siRNA showed comparably lower viability. This indicated that miR-130b negatively affected the viability of mesangial cells, whereas PTEN positively affected the viability of mesangial cells.

miR-130b and PTEN interfere with the apoptosis of mesangial cells. The present study subsequently investigated the relative apoptosis of mesangial cells when transfected with scramble control, miR-130b mimics, PTEN siRNA and miR-130b inhibitors. When transfected with miR-130b mimics and PTEN siRNA, the number of surviving mesangial cells was reduced and the number of apoptotic mesangial cells was increased, compared with numbers in the scramble control, whereas the mesangial cells transfected with miR-130b inhibitors showed comparably higher cell survival and reduced
A

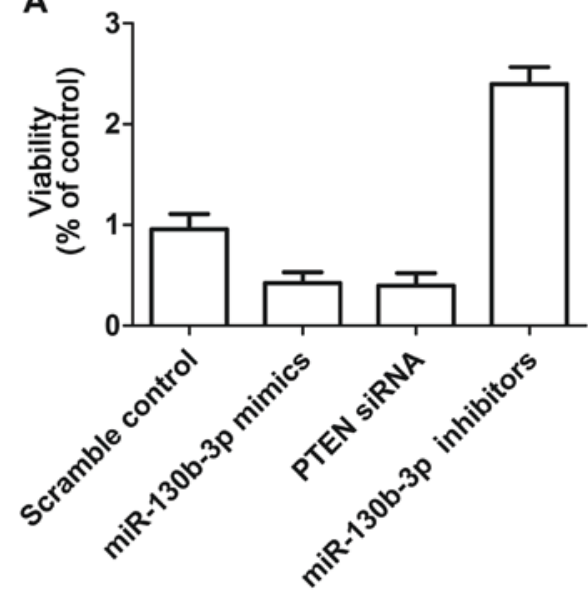

B

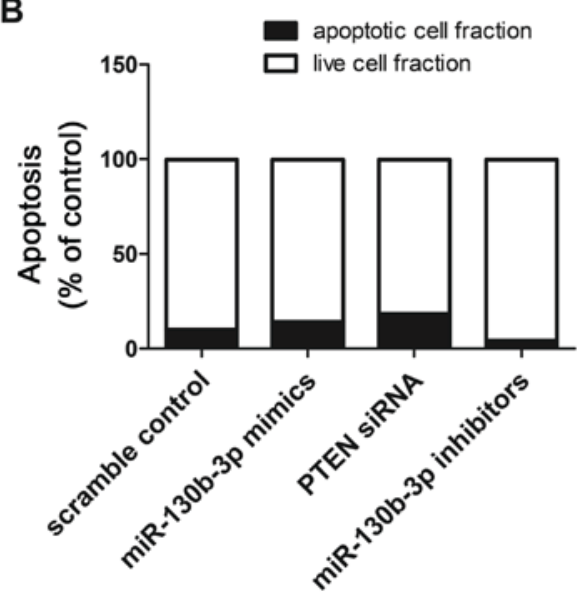

Figure 7. Effects on viability and apoptosis. (A) Mesangial cells transfected with miR-130b inhibitors showed upregulated viability, whereas mesangial cells transfected with miR-130b mimics and PTEN siRNA showed comparably lower viability. (B) Mesangial cells transfected with miR-130b mimics and PTEN siRNA showed accelerated apoptosis, whereas mesangial cells transfected with miR-130b inhibitors showed inhibited apoptosis. miR, microRNA; siRNA, small interfering RNA; PTEN, phosphatase and tensin homolog.

apoptotsis (Fig. 7B). These results indicated that miR-130b accelerated apoptosis, whereas PTEN inhibited apoptosis.

\section{Discussion}

MiRNAs are highly conserved small noncoding RNAs involved in numerous biologic processes. MiR-130b was reported to be associated with numerous diseases, including type 2 diabetes (22), colorectal cancer (23), and bladder cancer (24). Zhaohui Ni revealed that patients with early stage LN exhibited a higher level of miR-130b compared with healthy patients, and suggested that miR-130b may be involved in LN by regulating the expression of Erbb2 interacting protein (17). Xiao Han demonstrated that miR-130b was associated with pathogenesis of LN via regulating IRF-1 and further inhibited the type I IFN pathway (25). It is important to further investigate the mechanisms underlying miR-130b, mesangial cells and proteinuria in the future. A carefully designed investigation performed on a larger sample size is also necessary and the inclusion of other kidney conditions is 
likely to provide more valuable evidence. In the present study, kidney tissue samples were collected from patients with SLE, which were divided into two groups based on whether they had nephritis or not: Lupus nephritis(+) and lupus nephritis(-). RT-qPCR analysis was performed, and the results showed that the expression level of miR-130b was higher in the lupus nephritis(+) group (Fig. 1). In addition, online miRNA target prediction tools were used to identify the regulatory gene of miR-130b, and PTEN was identified as the candidate target gene of miR-130b with the 'seed sequence' in the 3'UTR (Fig. 2) and the existence of binding sites. A luciferase activity reporter assay was also performed on the mesangial cells, in which luciferase activity was only reduced in the mesangial cells cotransfected with miR-130b and wild-type PTEN 3'UTR (Fig. 3). The luciferase activities in mesangial cells cotransfected with miR-130b and mutant PTEN 3'UTR were comparable with that of the scramble control (Fig. 3).

PTEN is a potent tumor-inhibitor gene present at chromosome 10q23.31, which was identified in 1997 (26,27). A phosphatase with double properties against proteins and phospholipids is encoded by PTEN (28). The signal transduction pathways can be regulated by PTEN protein via either phosphatase-independent or dependent mechanisms (29). Regardless of its possible serine/threonine and tyrosine phosphatase property, the tumor-inhibitory effect of PTEN contributes to its lipid phosphatase effect (30). PTEN is considered to be the major factor negatively regulating class I PI3Ks (31). Additionally, the regulatory effect triggered by specific miRNAs on the function of important immune cells, including $\mathrm{B}$ and $\mathrm{T}$ lymphocytes, in lupus has been investigated. PTEN regulates normal signaling via the $\mathrm{B}$ cell receptor, and abnormal miR-7 regulation results in hyper-responsiveness of B cells in SLE (32). In the present study, a microarray was performed to determine the expression levels of miRNAs in B cells obtained from patients with active SLE, compared with healthy subjects. A marked reduction in the expression of miR-1246 expression in B cells was found in patients with active SLE, but not in patients with inactive SLE or healthy subjects, which suggested that miR-1246 was involved in active SLE and may offer potential as a biomarker or promising therapeutic target in active SLE. In the present study, it was found that the mRNA expression of PTEN (Fig. 5A) was decreased in the lupus nephritis(+) group, compared with that in the lupus nephritis(-) group. The protein expression of PTEN (Fig. 5B) was also measured using densitometric analysis, which revealed its expression was decreased in lupus nephritis(+), compared with that in lupus nephritis(-), and that the expression level of PTEN in mesangial cells treated with miR-130b mimics and PTEN siRNA were lower, compared with that in the scramble control. The expression of PTEN in mesangial cells treated with miR-130b inhibitors was higher than that in the scramble control, confirming the negative regulatory association between miR-130b and PTEN.

Apoptosis can occur via mitochondrial and receptor-mediated pathways. The importance of apoptosis in SLE has been confirmed, as apoptotic cells result in a high level of autoantigens, which appear to be processed inappropriately in this disease (33). Apoptotic suppression with a pan-caspase inhibitor or with anti-Fas ligand antibodies results in prevention against renal disorder in models of lupus nephritis $(34,35)$. It is increasingly accepted that inflammation and apoptosis can be correlated rather than being mutually exclusive (36). A previous observation that $\mathrm{C} 3 \mathrm{aRa}$ inhibited apoptosis and inflammatory cell infiltration is consistent with this and may even be caused by the same mechanism (37). The first step toward its membrane correlation and complete activation is the phosphorylation of PKB/Akt on serine 473, which was found to be substantially elevated by the inhibition of $\mathrm{C} 3 \mathrm{aR}$, possibly leading to the apoptotic decrease observed (38). Additionally, significantly higher levels of phosphorylated PTEN (serine 380) have been observed in control lupus mice, compared with that in C3aRa-treated mice; as PTEN negatively regulates PI $3 \mathrm{~K}$, and is closely associated with immune cell activation, cell growth and survival, these findings are likely to be correlated with the intrinsic pathophysiology of lupus nephritis and the mechanism by which signaling through C3aR causes disease $(39,40)$. In the present study, the relative apoptosis was investigated when of mesangial cells were transfected with scramble control, miR-130b mimics, PTEN siRNA and miR-130b inhibitors. When transfected with miR-130b mimics and PTEN siRNA, the number of surviving mesangial cells was reduced and the number of apoptotic mesangial cells was increased, compared with those in the scramble controls group, whereas the mesangial cells transfected with miR-130b inhibitors showed comparably higher numbers of surviving cells and fewer apoptotic mesangial cells.

In conclusion, the present study demonstrated that miR-130b was upregulated in the lupus nephritis group, compared with that in the control group. PTEN was identified as a virtual target of miR-130b, and there was a negative regulatory association between miR-130b and PTEN. miR-130b and PTEN interfered with the viability and apoptosis of mesangial cells.

\section{Acknowledgements}

Not applicable.

\section{Funding}

No funding was received.

\section{Availability of data and materials}

All data generated or analyzed during this study are included in this published article.

\section{Authors' contributions}

SW planned the study, collected the data, analysed and interpreted the data and prepared the manuscript. JW collected the data, prepared the manuscript. FL interpreted the data, collected the literature.

\section{Ethics approval and consent to participate}

The present study was approved by the Ethics Committee of the Hospital of Tai'an Central Hospital. Written consent was signed by all patients. 


\section{Consent for publication}

Written consent was signed by all patients for the publication of any associated data or accompanying images.

\section{Competing interests}

The authors declare that they have no competing interests.

\section{References}

1. Helmick CG, Felson DT, Lawrence RC, Gabriel S, Hirsch R Kwoh CK, Liang MH, Kremers HM, Mayes MD, Merkel PA, et al: Estimates of the prevalence of arthritis and other rheumatic conditions in the United States. Part I. Arthritis Rheum 58: 15-25, 2008

2. Peng SL: Altered T and B lymphocyte signaling pathways in lupus. Autoimmun Rev 8: 179-183, 2009.

3. Hogan $\mathrm{J}$ and Radhakrishnan $\mathrm{J}$ : The treatment of minimal change disease in adults. J Am Soc Nephrol 24: 702-711, 2013.

4. Bertsias G, Gordon C and Boumpas DT: Clinical trials in systemic lupus erythematosus (SLE): Lessons from the past as we proceed to the future-the EULAR recommendations for the management of SLE and the use of end-points in clinical trials. Lupus 17: 437-442, 2008

5. Ceman S and Saugstad J: MicroRNAs: Meta-controllers of gene expression in synaptic activity emerge as genetic and diagnostic markers of human disease. Pharmacol Ther 130: 26-37, 2011.

6. Lorenzen JM, Haller H and Thum T: MicroRNAs as mediators and therapeutic targets in chronic kidney disease. Nat Rey Nephrol 7: 286-294, 2011.

7. Farris $\mathrm{AB}$ and Colvin RB: Renal interstitial fibrosis: Mechanisms and evaluation. Curr Opin Nephrol Hypertens 21: 289-300, 2012

8. Wang G, Kwan BC, Lai FM, Choi PC, Chow KM, Li PK and Szeto CC: Intrarenal expression of miRNAs in patients with hypertensive nephrosclerosis. Am J Hypertens 23: 78-84, 2010.

9. Wang G, Kwan BC, Lai FM, Choi PC, Chow KM, Li PK and Szeto CC: Intrarenal expression of microRNAs in patients with IgA nephropathy. Lab Invest 90: 98-103, 2010.

10. Chung AC, Huang XR, Meng X and Lan HY: miR-192 mediates TGF-beta/Smad3-driven renal fibrosis. J Am Soc Nephrol 21: 1317-1325, 2010.

11. Kato M, Zhang J, Wang M, Lanting L, Yuan H, Rossi JJ and Natarajan R: MicroRNA-192 in diabetic kidney glomeruli and its function in TGF-beta-induced collagen expression via inhibition of E-box repressors. Proc Natl Acad Sci USA 104: 3432-3437, 2007.

12. Putta S, Lanting L, Sun G, Lawson G, Kato M and Natarajan R: Inhibiting microRNA-192 ameliorates renal fibrosis in diabetic nephropathy. J Am Soc Nephrol 23: 458-469, 2012.

13. Dai Y, Sui W, Lan H, Yan Q, Huang H and Huang Y: Comprehensive analysis of microRNA expression patterns in renal biopsies of lupus nephritis patients. Rheumatol Int 29: 749-754, 2009

14. Lu J, Kwan BC, Lai FM, Tam LS, Li EK, Chow KM, Wang G, Li PK and Szeto CC: Glomerular and tubulointerstitial miR-638, miR-198 and miR-146a expression in lupus nephritis. Nephrology (Carlton) 17: 346-351, 2012

15. Rabinovitch MB Liberman and Fausto N: Plasma ribonuclease activity in human uremia. J Lab Clin Med 53: 563-568, 1959.

16. Neal CS, Michael MZ, Pimlott LK, Yong TY,Li JY and Gleadle JM: Circulating microRNA expression is reduced in chronic kidney disease. Nephrol Dial Transplant 26: 3794-3802, 2011.

17. Wang W, Mou S, Wang L, Zhang M, Shao X, Fang W, Lu R, Qi C, Fan Z, Cao Q, et al: Up-regulation of serum MiR-130b-3p level is associated with renal damage in early lupus nephritis. Sci Rep 5: 12644, 2015

18. Tan W, Gu Z, Shen B, Jiang J, Meng Y, Da Z, Liu H, Tao T and Cheng C: PTEN/Akt-p27(kip1) signaling promote the BM-MSCs senescence and apoptosis in SLE patients. J Cell Biochem 116: $1583-1594,2015$

19. Tan EM, Cohen AS, Fries JF, Masi AT, McShane DJ, Rothfield NF, Schaller JG, Talal N and Winchester RJ: The 1982 revised criteria for the classification of systemic lupus erythematosus. Arthritis Rheum 25: 1271-1277, 1982.
20. Lamb EJ, Webb MC and O'Riordan SE: Using the modification of diet in renal disease (MDRD) and Cockcroft and Gault equations to estimate glomerular filtration rate (GFR) in older people. Age Ageing 36: 689-692, 2007.

21. Livak KJ and Schmittgen TD: Analysis of relative gene expression data using real-time quantitative PCR and the 2(-Delta Delta $\mathrm{C}(\mathrm{T})$ ) method. Methods 25: 402-408, 2001.

22. Lv C, Zhou YH, Wu C, Shao Y, Lu CL and Wang QY: The changes in miR-130b levels in human serum and the correlation with the severity of diabetic nephropathy. Diabetes Metab Res Rev 31: 717-724, 2015.

23. Colangelo T, Fucci A, Votino C, Sabatino L, Pancione M, Laudanna C, Binaschi M, Bigioni M, Maggi CA, Parente D, et al: MicroRNA-130b promotes tumor development and is associated with poor prognosis in colorectal cancer. Neoplasia 15: 1086-1099, 2013.

24. Egawa H, Jingushi K, Hirono T, Ueda Y, Kitae K, Nakata W, Fujita K, Uemura M, Nonomura N and Tsujikawa K: The miR-130 family promotes cell migration and invasion in bladder cancer through FAK and Akt phosphorylation by regulating PTEN. Sci Rep 6: 20574, 2016.

25. Han X, Wang Y, Zhang X, Qin Y, Qu B, Wu L, Ma J, Zhou Z, Qian J, Dai M, et al: MiR-130b ameliorates murine lupus nephritis through targeting type I interferon pathway on resident renal cells. Arthritis Rheumatol 68: 2232-2243, 2016.

26. Govender D and Chetty R: Gene of the month: PTEN. J Clin Pathol 65: 601-603, 2012.

27. Li DM and Sun H: TEP1, encoded by a candidate tumor suppressor locus, is a novel protein tyrosine phosphatase regulated by transforming growth factor beta. Cancer Res 57: 2124-2129, 1997.

28. Di Cristofano A and Pandolfi PP: The multiple roles of PTEN in tumor suppression. Cell 100: 387-390, 2000.

29. Xu WT, Yang Z and Lu NH: Roles of PTEN (Phosphatase and Tensin Homolog) in gastric cancer development and progression. Asian Pac J Cancer Prev 15: 17-24, 2014.

30. Myers MP and Tonks NK: PTEN: Sometimes taking it off can be better than putting it on. Am J Hum Genet 61: 1234-1238, 1997.

31. Ye B, Jiang LL, Xu HT, Zhou DW and Li ZS: Expression of PI3K/AKT pathway in gastric cancer and its blockade suppresses tumor growth and metastasis. Int J Immunopathol Pharmacol 25: 627-636, 2012

32. Wu XN, Ye YX, Niu JW, Li Y, Li X, You X, Chen H, Zhao LD, Zeng XF, Zhang FC, et al: Defective PTEN regulation contributes to B cell hyperresponsiveness in systemic lupus erythematosus. Sci Transl Med 6: 246ra99, 2014.

33. Kaplan MJ: Apoptosis in systemic lupus erythematosus. Clin Immunol 112: 210-218, 2004.

34. Seery JP, Cattell V and Watt FM: Cutting edge: Amelioration of kidney disease in a transgenic mouse model of lupus nephritis by administration of the caspase inhibitor carbobenzoxy-valyl-alan yl-aspartyl-(beta-o-methyl)-fluoromethylketone. J Immunol 167: 2452-2455, 2001

35. Nakajima A, Hirai H, Kayagaki N, Yoshino S, Hirose S, Yagita $H$ and Okumura K: Treatment of lupus in NZB/W F1 mice with monoclonal antibody against Fas ligand. J Autoimmun 14: 151-157, 2000.

36. Schaub FJ, Han DK, Liles WC, Adams LD, Coats SA, Ramachandran RK, Seifert RA, Schwartz SM and Bowen-Pope DF: Fas/FADD-mediated activation of a specific program of inflammatory gene expression in vascular smooth muscle cells. Nat Med 6: 790-796, 2000.

37. Lenda DM, Kikawada E, Stanley ER and Kelley VR: Reduced macrophage recruitment, proliferation, and activation in colony-stimulating factor-1-deficient mice results in decreased tubular apoptosis during renal inflammation. J Immunol 170: 3254-3262, 2003

38. Scheid MP and Woodgett JR: Unravelling the activation mechanisms of protein kinase B/Akt. FEBS Lett 546: 108-112, 2003.

39. Deane JA and Fruman DA: Phosphoinositide 3-kinase: Diverse roles in immune cell activation. Annu Rev Immunol 22: 563-598, 2004.

40. Cantley LC and Neel BG: New insights into tumor suppression: PTEN suppresses tumor formation by restraining the phosphoinositide 3-kinase/AKT pathway. Proc Natl Acad Sci USA 96: 4240-4245, 1999. 